\title{
Characterization and expression pattern analysis of the facilitative glucose transporter 10 gene (s/c2a10) in Danio rerio
}

\author{
NICOLA CHIARELLI, MARCO RITELLI, NICOLETTA ZOPPI, ANNA BENINI, GIUSEPPE BORSANI, \\ SERGIO BARLATI and MARINA COLOMBI* \\ Division of Biology and Genetics, Department of Biomedical Sciences and Biotechnology, \\ Medical Faculty, University of Brescia, Brescia, Italy.
}

\begin{abstract}
The SLC2A10 gene located on chromosome 20q13.1 encodes the facilitative glucose transporter 10 (GLUT10), a class III member of the SLC2A facilitative glucose transporter family. Mutations in the human SLC2A10 gene cause arterial tortuosity syndrome (ATS), a rare autosomal recessive connective tissue disorder. In this work, we report the characterization of the slc2a10 ortholog gene in zebrafish (Danio rerio) and its expression pattern during embryonic development and in adult tissues. The slc2a10 gene consists of 5 exons, spanning 8 kb and mapping to a region on chromosome 11 that exhibits conserved synteny with human chromosome 20. The gene encodes Glut10, a 513 amino acid protein that maintains the 12 transmembrane domain structure typical of the GLUTs family, and shares the specific functional motifs involved in sugar transport with the vertebrate GLUT10. RT-PCR analysis showed that two specific splice variants, both including the $5^{\prime}$-UTR region, were expressed during embryogenesis and in different adult zebrafish tissues and organs. In situ hybridization analyses demonstrated a maternal origin of the total s/c2a10 mRNA and its ubiquitous distribution until the early somitogenesis stage. In later embryonic stages, slc2a10 mRNA was detected in the otic vesicles, hatching gland cells, pectoral fin, posterior tectum and swim bladder. Overall, these results suggest a wide role of slc2a10 during zebrafish development.
\end{abstract}

KEY WORDS: slc2a10, Glut10, facilitative glucose transporter, arterial tortuosity syndrome

The transport of glucose and hexoses across the plasma membrane is mediated by a family of facilitative glucose transporters ( $S L C 2 A$ gene, GLUT protein). In the mammalian genome, 14 different GLUT isoforms with distinct kinetic properties of transport and tissue/cell-specific expression patterns have been described (Zhao and Keating, 2007). These sugar transporters are integral membrane proteins containing 12 transmembrane hydrophobic segments, which are connected by 6 extracellular and 5 intracellular loops with both amino and carboxyl-termini exposed in the cytosol. On the basis of amino acid sequence similarities and transport characteristics, the mammalian GLUTs are grouped into three classes: class I, (GLUT1-4 and GLUT14), class II (GLUT5-7-9 and 11) and class III (GLUT6, 8, 10, 12 and the $\mathrm{H}^{+}$coupled myo-inositol transporter, HMIT) (Joost and Thorens, 2001; Joost et al., 2002; Uldry and Thorens, 2004). Human
GLUT10 has been shown to facilitate D-glucose, D-galactose and 2-deoxy-D-glucose uptake when expressed in Xenopus laevis oocytes (Dawson et al., 2001). GLUT10 mRNA is expressed at

Abbreviations used in this paper: AA, ascorbic acid; ATS, arterial tortuosity syndrome; BCIP, bromo-4-chloro-3-indolyl phosphate; cds, coding sequence; DIG, digoxygenin; dpf, days post fertilization; EDTA, ethylenediaminetetra-acetic acid; GLUTs, facilitative glucose transporter family; GLUT10, facilitative glucose transporter member 10; hpf, hours post-fertilization; ISH, in situ hybridization; NBT, nitro blue tetrazolium; PCR, polymerase chain reaction; Q-PCR, quantitative real-time PCR, RTPCR, reverse-transcription PCR; SLC2A, solute carrier family 2; SLC2A10, solute carrier family 2 member 10; SNP, single-nucleotide polymorphism; TBE, tris base-boric acid-EDTA; TMD, transmembrane domain; UTR, untranslated region.

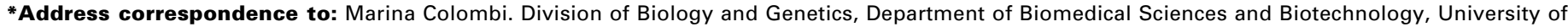
Brescia, Viale Europa 11, 25123 Brescia, Italy. Fax: +39-030-370-1157. e-mail: colombi@med.unibs.it web: http://www.unibs.it/on-line/med/Home/Docenti/scheda99.html
}

Supplementary Material (a Table) for this paper is available at: http://dx.doi.org/10.1387/ijdb.103179nc 
highest levels in the liver and pancreas, but is also expressed in the heart, lung, brain, skeletal muscle, placenta and adipose tissue (Dawson et al., 2001; McVie-Wylie et al., 2001). In vitro, $S L C 2 A 10$ is expressed by cultured vascular smooth muscle cells (VSMC) and skin fibroblasts, which localize the protein either to the plasma membrane or the perinuclear envelope (Coucke etal., 2006). Recently, it has been proposed that GLUT10 may transport ascorbic acid (AA), a cofactor for collagen and elastin synthesis, to the secretory pathway (Segade, 2010). In particular, the author demonstrated that GLUT10 localizes to the endoplasmic reticulum. Moreover, Lee et al. (2010) showed that GLUT10 also localizes to the mitochondria of smooth muscle cells, facilitating transport and uptake of AA into these organelles, which in turn protects cells against oxidative stress.

Loss-of-function mutations in the $S L C 2 A 10$ gene lead to arterial tortuosity syndrome (ATS) (OMIM \#208050) (Coucke et al., 2006; Drera et al., 2007, Callewaert et al., 2008a, Faiyaz-UIHaque et al., 2009; Zaidi et al., 2009; Ritelli et al., 2009), a rare autosomal recessive connective tissue disorder characterized by tortuosity and elongation of the large and medium sized arteries, a propensity for aneurysm formation, vascular dissection, and pulmonary artery stenosis. Two mouse models for ATS were proposed, but they did not fully mimic the human syndrome, and the reported phenotypes of these mice remain inconclusive with respect to GLUT10 function (Callewaert et al., 2008b, Cheng et al., 2009). In fact, the role of this transporter in the etiology of ATS is still controversial (Coucke et al., 2006; Akhurst, 2006; Segade, 2010; Lee et al., 2010).

The teleost fish Danio rerio has become an important animal model for studies of vertebrate development as well as the molecular bases of human diseases (Parant et al., 2010). Recently, 18 members of the $S L C 2 A$ family were identified in this experimental model, including the zebrafish ortholog of the human SLC2A10 gene (Tseng et al., 2009).

In this study, we report a more detailed characterization of the s/c2a10 gene; in particular, we identified two different s/c2a10 splice variants, defined their spatiotemporal expression pattern during embryogenesis by quantitative real-time PCR and wholemount in situ hybridization (ISH) and characterized the specific distribution of s/c2a 10 mRNA in several adult zebrafish tissues by RT-PCR.
TABLE 1

\section{SNPs IDENTIFIED IN THE sIc2a10 CODING REGION}

\begin{tabular}{|c|c|c|c|c|c|c|}
\hline Exon $n^{\circ}$ & $\begin{array}{l}\text { Position } \\
\text { (cds) }\end{array}$ & Substitution & SNP & aa codon & $\begin{array}{l}\text { Codon } \\
\text { position }\end{array}$ & $\begin{array}{c}\text { aa } \\
\text { position }\end{array}$ \\
\hline 2 & 78 & synonymous & ATA/T & Ile (I)/lle (I) & 3 & 26 \\
\hline 2 & 252 & synonymous & $\mathrm{CTG} / \mathrm{A}$ & Leu (L)/Leu (L) & 3 & 84 \\
\hline 2 & 253 & non-synonymous & G/ACC & Ala $(\mathrm{A}) / \mathrm{Thr}(\mathrm{T})$ & 1 & 85 \\
\hline 2 & 306 & synonymous & $\mathrm{GGC} / \mathrm{T}$ & Gly (G)/Gly (G) & 3 & 102 \\
\hline 2 & 307 & synonymous & $A / C G G$ & $\operatorname{Arg}(R) / \operatorname{Arg}(R)$ & 1 & 103 \\
\hline 2 & 315 & synonymous & $\mathrm{GTG} / \mathrm{T}$ & Val (V)/Val (V) & 3 & 105 \\
\hline 2 & 420 & synonymous & GGG/A & Gly (G)/Gly (G) & 3 & 140 \\
\hline 2 & 421 & non-synonymous & A/GTC & Ile (I)/Val (V) & 1 & 141 \\
\hline 2 & 447 & synonymous & TAT/C & $\operatorname{Tyr}(\mathrm{Y}) / \operatorname{Tyr}(\mathrm{Y})$ & 3 & 149 \\
\hline 2 & 450 & synonymous & GCT/GCC & $\mathrm{Ala}(\mathrm{A}) / \mathrm{Ala}(\mathrm{A})$ & 3 & 150 \\
\hline 2 & 460 & non-synonymous & T/ATC & Phe(F)/lle(I) & 1 & 154 \\
\hline 2 & 474 & synonymous & GTG/A & Val (V)/Val (V) & 3 & 158 \\
\hline 2 & 476 & non-synonymous & $\mathrm{CA} / \mathrm{CA}$ & $\operatorname{Gln}(\mathrm{Q}) / \operatorname{Pro}(\mathrm{P})$ & 2 & 159 \\
\hline 2 & 641 & non-synonymous & CG/AT & $\operatorname{Arg}(\mathrm{R}) / \mathrm{His}(\mathrm{H})$ & 2 & 214 \\
\hline 2 & 711 & synonymous & GTG/A & Val (V)/Val (V) & 3 & 237 \\
\hline 2 & 730 & synonymous & C/TTG & Leu (L)/Leu (L) & 1 & 244 \\
\hline 2 & 768 & synonymous & T/CTA & Leu (L)/Leu (L) & 1 & 256 \\
\hline 2 & 903 & synonymous & $A G A / G$ & $\operatorname{Arg}(\mathrm{R}) / \operatorname{Arg}(\mathrm{R})$ & 3 & 301 \\
\hline 2 & 910 & synonymous & C/TTG & Leu (L)/Leu (L) & 1 & 304 \\
\hline 2 & 968 & non-synonymous & $\mathrm{AG} / \mathrm{CA}$ & $\operatorname{Arg}(\mathrm{R}) / \operatorname{Thr}(\mathrm{T})$ & 2 & 323 \\
\hline 2 & 1.047 & non-synonymous & $\mathrm{GAA} / \mathrm{C}$ & Glu(E)/Asp(D) & 3 & 349 \\
\hline 3 & 1.263 & synonymous & $\mathrm{TTC} / \mathrm{T}$ & Phe (F)/Phe (F) & 3 & 421 \\
\hline 3 & 1.273 & non-synonymous & $\mathrm{G} / \mathrm{ACA}$ & Ala $(\mathrm{A}) / \mathrm{Thr}(\mathrm{T})$ & 1 & 425 \\
\hline 3 & 1.289 & non-synonymous & $\mathrm{AC} / \mathrm{GC}$ & $\operatorname{Thr}(\mathrm{T}) / \mathrm{Ser}(\mathrm{S})$ & 2 & 430 \\
\hline 4 & 1.497 & synonymous & $\mathrm{TTT} / \mathrm{C}$ & Phe(F)/Phe(F) & 3 & 499 \\
\hline
\end{tabular}

All synonymous and non-synonymous substitutions are defined using as reference sequence the s/c2a10 coding sequence (accession nos.: HM560591 and HM560592) obtained in this study, compared to those present in GenBank (accession nos.: XM 679477.2 and NM 001111163). The nucleotides are numbered starting from the first base of the start codon (ATG) of the s/c2a10 coding sequence. aa, amino acid; cds, coding sequence; SNP, single nucleotide polymorphism.

\section{Results}

\section{Characterization of slc2a10 in zebrafish}

To identify the zebrafish ortholog of the human SLC2A10 gene, the sequence of human GLUT10 protein was used as a query in BLAT searches against the Zebrafish genome sequence using the UCSC Genome Browser. This analysis led to the identification of the genomic locus LOC560546, predicted to

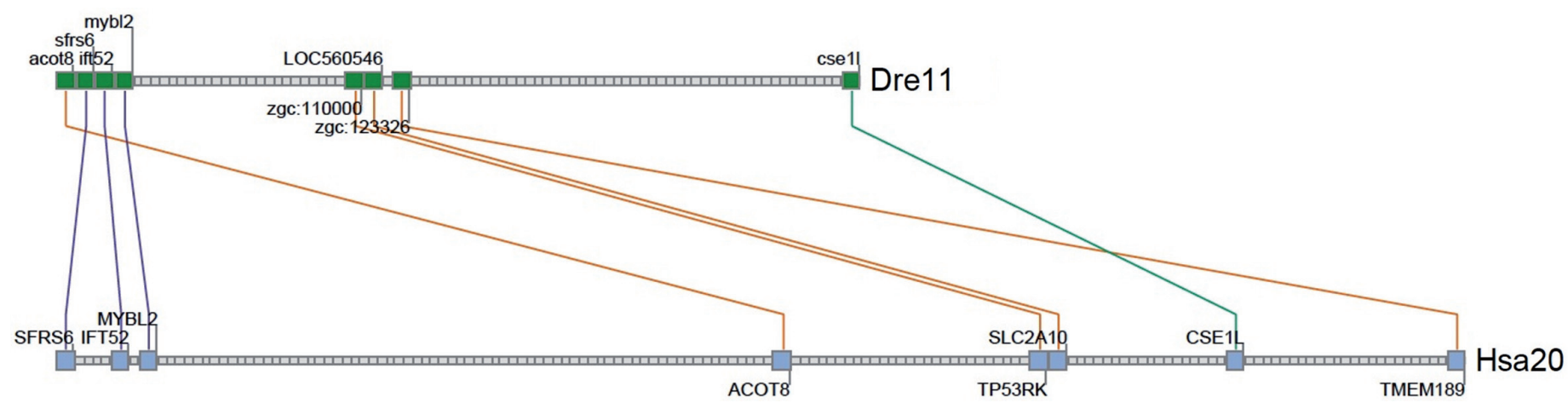

Fig. 1. Graphical representation of conserved synteny neighboring the SLC2A10 locus between $D$. rerio chromosome 11 (Dre11) and $H$. sapiens chromosome 20 (Hsa20). The gene trace representation was generated using the Synteny DB. Genes are drawn as squares with their order but without preservation of their physical location. Lines connecting squares between the two clusters represent orthologous gene pairs. Lines of the same color, referred to as subclusters, generally indicate strong local conservation. The analysis was performed on D. rerio Sanger Zv7 and $\mathrm{H}$. sapiens NCBI v36 genome assemblies with a sliding window size of 100 genes. 
TABLE 2

\section{EXON-INTRON ORGANIZATION OF THE sIc2a10 GENE}

\begin{tabular}{|c|c|c|c|c|c|}
\hline \multirow[b]{2}{*}{ Exon $\mathrm{n}^{\circ}$} & \multirow[b]{2}{*}{$\begin{array}{c}\text { Exon size } \\
\text { (bp) }\end{array}$} & \multicolumn{2}{|c|}{ Sequence at exon-intron junction } & \multirow[b]{2}{*}{$\begin{array}{l}\text { Intron size } \\
\text { (bp) }\end{array}$} & \multirow[b]{2}{*}{$\begin{array}{l}\text { Amino acid } \\
\text { interrupted }\end{array}$} \\
\hline & & 5' splice donor & 3' splice acceptor & & \\
\hline 1 & $>400$ & AAAATGGgtaagttg & tgctccagGTTGTTCT & 2.391 & Gly-2 \\
\hline 2 & 1.185 & GGACCAAgtaagtgg & gtttgacag TGACGTG & 1.286 & Met-397 \\
\hline 3 & 123 & ATAATCGgtgtgcac & tcttctcagATGTCATT & 93 & Asp-438 \\
\hline 4 & 136 & AGACACGgtaagagg & ttctttcagAATGATC & 1.950 & Arg-483 \\
\hline 5 & 202 & & & & \\
\hline
\end{tabular}

Exon sequences are indicated by uppercase letters and intron sequences by lowercase letters. Splice donor and acceptor sites are underlined. Exon and intron size are reported as base pairs. The corresponding interrupted amino acid residue in the Glut10 protein is also reported.

encode for a protein similar to human GLUT10, on chromosome 11 of the zebrafish genome. This putative encoded protein shows a high degree of amino acid sequence similarity $(43 \%)$ with human GLUT10. Furthermore, analysis of an approximately $5 \mathrm{Mb}$ genomic region surrounding the $S L C 2 A 10$ gene in zebrafish and in humans showed the presence of conserved synteny between this region of Danio rerio chromosome 11 and Homo sapiens chromosome 20q13.12. In both species, the gene is flanked on its 5' side by the TP53RK gene and more distantly by the ACOT8, MYBL2, IFT52 and SFRS6 genes. On the 3' side, the CSE1L and TMEM189 genes can be identified (Fig. 1). These data highly suggest that the genomic locus LOC560546 contains the ortholog of the human $S L C 2 A 10$ gene, which is named s/c2a10 (protein Glut10) according to the Zebrafish Nomenclature Guidelines.

Using the BLAST algorithm on the dbEST database, we identified several entries corresponding to s/c2a10 gene transcripts. We selected two putative full-length IMAGE clones, no. 5601920 and no. 7225584, which were completely sequenced. This analysis revealed a 24-bp insertion that contains a stop codon in IMAGE clone 7225584, whereas clone no. 5601920 contains an entire ORF (1.542 bp, 513 aa). Furthermore, sequence analysis showed that these two clones differ in their 5' UTR region, suggesting the presence of two splice variants. Recently, Tseng et al. (2009) identified eighteen members of glucose transporters in zebrafish, including s/c2a10 (deposited mRNA sequence NM_001111163). Using the GenBank entry XM_679477.2 generated using a gene prediction method and the sequence of IMAGE clone no. 5601920, we performed multiple amino acid alignments that led to the identification of seven amino

Fig. 2. Temporal expression pattern analysis of s/c2a10 during development and in adult zebrafish tissues. RNA samples were purified from different embryonic and larval stages and from adult fishes and amplified with specific primer pairs of slc2a10. RT-PCR analysis (A) showed that two splice variants were expressed both maternally and at different developmental stages, similar to adult fish. Q-PCR (B) was used to quantify the expression levels of total slc2a10 mRNA during embryogenesis and in adult fishes. Expression of ef- $1 \alpha$ housekeeping gene was used to normalize the relative expression levels of slc2a10 mRNA, which were compared to the corresponding values of the 1-cell stage (set to 1). Two specific PCR products of 727 and 550 bp were amplified from total RNA purified from adult zebrafish liver, heart, brain, eyes, gills, spleen, ovary, swim bladder, caudal fins, intestine, testes and kidney (C). To assess the RNA quality, a $\beta$-actin mRNA-specific product was obtained from all tissues analyzed. Abbreviations: $A$, adult; $M$, Size Marker. acid differences among the three sequences (not shown). Evolutionary conservation analyses, performed by multiple ClustalW alignment of several GLUT10 orthologs (H. sapiens, M. muscuIus, $R$. norvegicus, $G$. gallus and $X$. tropicalis) suggested that six out of these seven missense substitutions should be considered polymorphic variants. The highly conserved lysine at position 284 in the transmembrane domain 8 (TMD8) of the protein was the exception, having changed to a glutamic acid in the IMAGE clone (not shown). In order to clarify these discrepancies, we decided to re-clone and sequence the s/c2a10 cds using an RT-PCR strategy performed on adult zebrafish RNA. This nucleotide sequence was used as internal reference sequence and deposited in the GenBank database (accession nos.: HM560591 and HM560592). In addition, to validate this sequence, the cds was amplified and sequenced from total RNAs purified from different adult fishes and a pool of embryos. The ClustalW multiple sequence alignment of all these sequences and those deposited in GenBank (accession nos: XM_679477 and NM_001111163) revealed a total of 25 SNPs in the s/c2a10 coding region, nine of which are missense substitutions (Table 1). These nucleotide differences are likely due to high genetic variation existing in the different zebrafish strains (Guryev et al., 2006). Moreover, we did not find the p.K284E variant identified in clone no. 5601920 . This substitution could be a mutational event, which probably arose during cloning, similar to the 24-bp insertion identified in clone no. 7225584

The 5' and 3' UTRs of s/c2a 10 were obtained using a combined strategy based on computational analyses and sequencing of IMAGE clones and RT-PCR fragments of s/c2a10 cDNAs. As suggested by the sequencing of the IMAGE clones, we identified two mRNA splice variants in the 5'-UTR, which were expressed both during embryogenesis and in adult zebrafish tissues (Fig. 2A and $2 \mathrm{C}$ ). In particular, we characterized a longer isoform with a

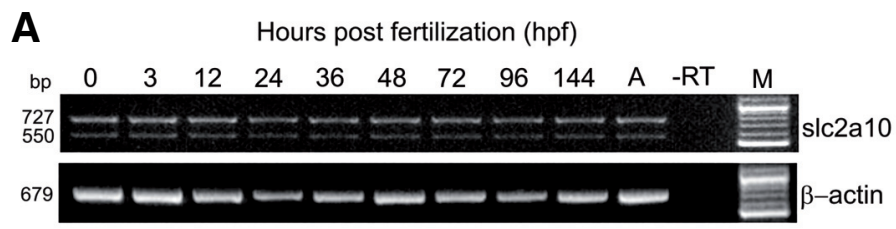

B
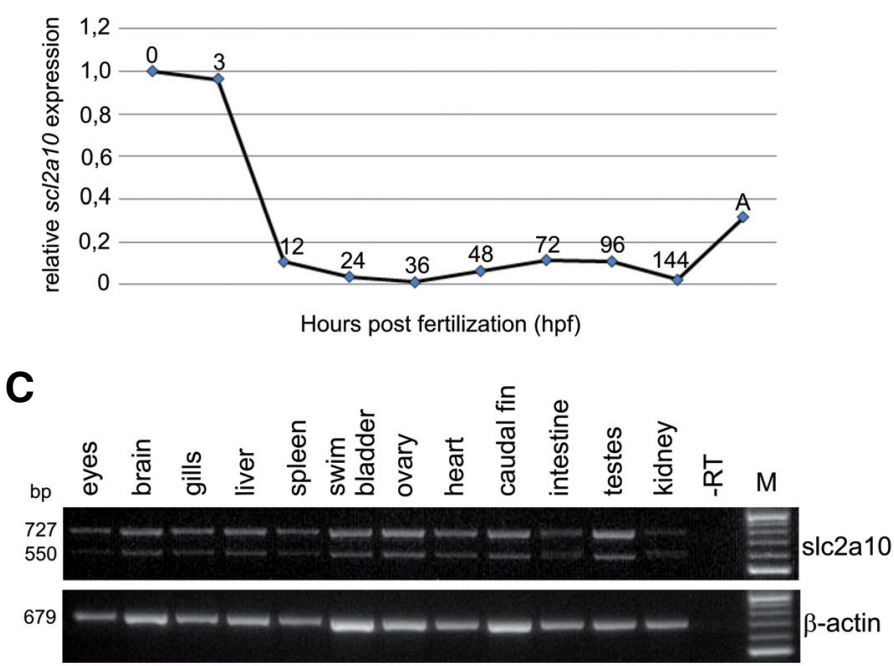


\section{N. Chiarelli et al.}

177-nucleotide stretch and a shorter variant without this nucleotide sequence. Both splice variants were deposited in GenBank with the accession numbers: HM560591 (+ 177 splice form) and HM560592 (-177 splice form).

The s/c2a10 gene is distributed among five exons, which is the same as the human counterpart, and the intron-exon splice junctions are conserved within the two species (Table 2). The Glut10 protein was compared to vertebrate proteins from $H$. sapiens, M. musculus, $R$. norvegicus, G. gallus and $X$. tropicalis (Fig. 3). Multiple sequence alignment revealed that zebrafish Glut10 is $45 \%$ identical to human GLUT10, 44\% identical to $M$. musculus and $R$. norvegicus orthologs and shares $48 \%$ sequence identity to $G$. gallus and X. tropicalis GLUT10. Furthermore, Glut10 maintains the typical 12 hydrophobic TMD structure and structural motifs involved in glucose transport activity (Fig. 3). In particular, zebrafish Glut10, as in the human and the mouse counterparts, retains the tryptophan residue $\mathrm{W}^{399}$ in TMD10 and the glutamic acid $E^{404}$ and glycine $\mathrm{G}^{412}$ residues in intracellular loop 5 (Fig. 3). These residues are important for substrate recognition and sugar transport specificity (Barrett et al., 1999; McVieWylie et al., 2001; Dawson et al., 2001). Interestingly, zebrafish Glut10 does not have the PESPR motif in loop 6 present in all GLUTs, which may contribute to its low-rate saturable glucose transport activity (Dawson et al., 2001; Zhao and Keating, 2007).

\section{Temporal expression pattern of the slc2a10 gene}

To investigate the temporal expression pattern of s/c2a10 during zebrafish development, we performed both RT-PCR and quantitative PCR (Q-PCR) analyses on cDNA obtained from

Fig. 3. Multiple sequence alignment and amino acids sequence identity of Glut10 protein with GLUT10 orthologous proteins in various species. The deduced amino acids sequence of Glut10 was compared to GLUT10 sequences from $\mathrm{H}$. sapiens (NP_110404), M. musculus (NP_569718), R norvegicus (NP_001102433), G. gallus (predicted, $\left.X P \_417383.2\right)$, and $X$. tropicalis (AA_22090). The zebrafish protein shares $45 \%$ sequence identity with the human GLUT10 and maintains the typical 12 TMD structure, as indicated by the dashed lines on the top of the alignment. The signature sequence motifs involved in glucose transport property are reported on the bottom of the protein sequence alignment. Black and gray boxes indicate identity and similarity between the amino acid sequences, respectively. White boxes indicate non-conserved amino acid substitutions. Multiple alignment was performed with ClustalW software and analyzed by GeneDoc program.
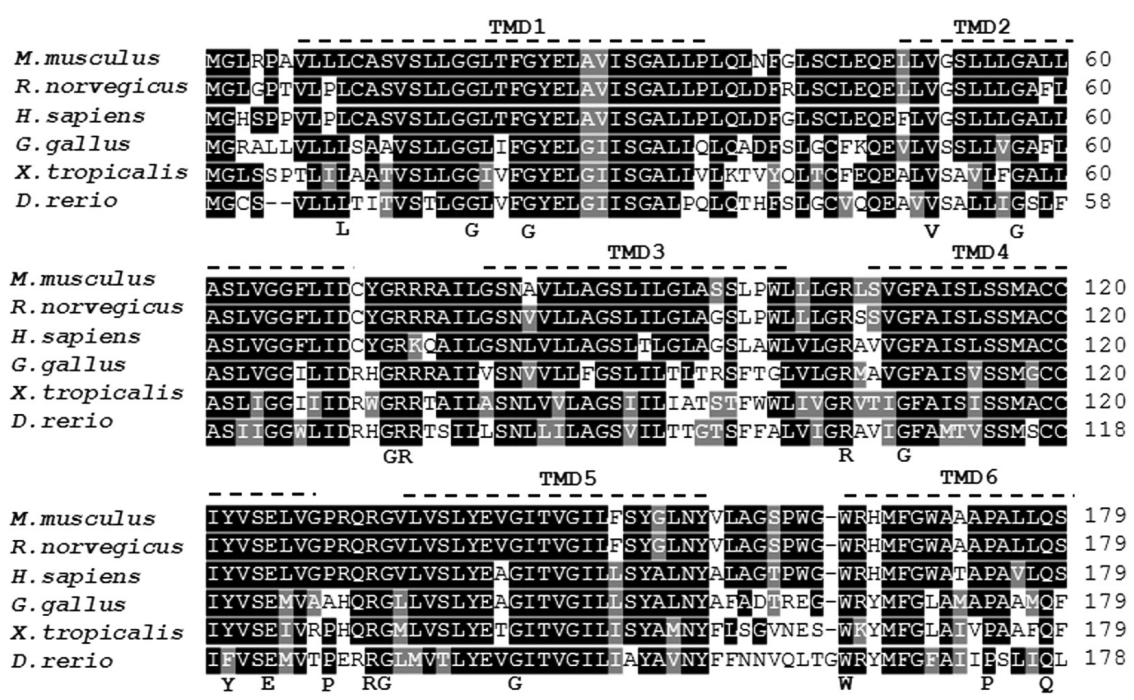

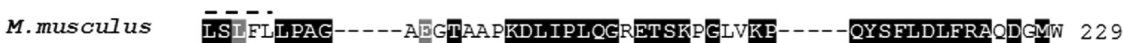
R. norvegicus LSLFFLPAG----AFG TAARDLIPLQGGETSKLGLVkA----QYYFLDLFRA QDGMW 229 H.sapiens LSLLFLPAG----TDETATHKDIPLQGGEAPKLGPGRE----RYSFLDLFRARDNMR 229 G.gallus LSILFLEVNPVKLNTWDSDCQKGGLIOLOTAEDREAARRE EYKEK-HYSFLDLFRTRDNMR 238 X. tropicalis ISILFLFSRPHKLNFWËQDDDGFIELE--ETGEAGEFREDTYDRQYTFLDLFRSKDNMR 237 D. rerio ASIVLLEKQAEVFVIHDDDSRQADRLTEETETSNQRQQSE-----KYGVSDLFKKKLNMR 233
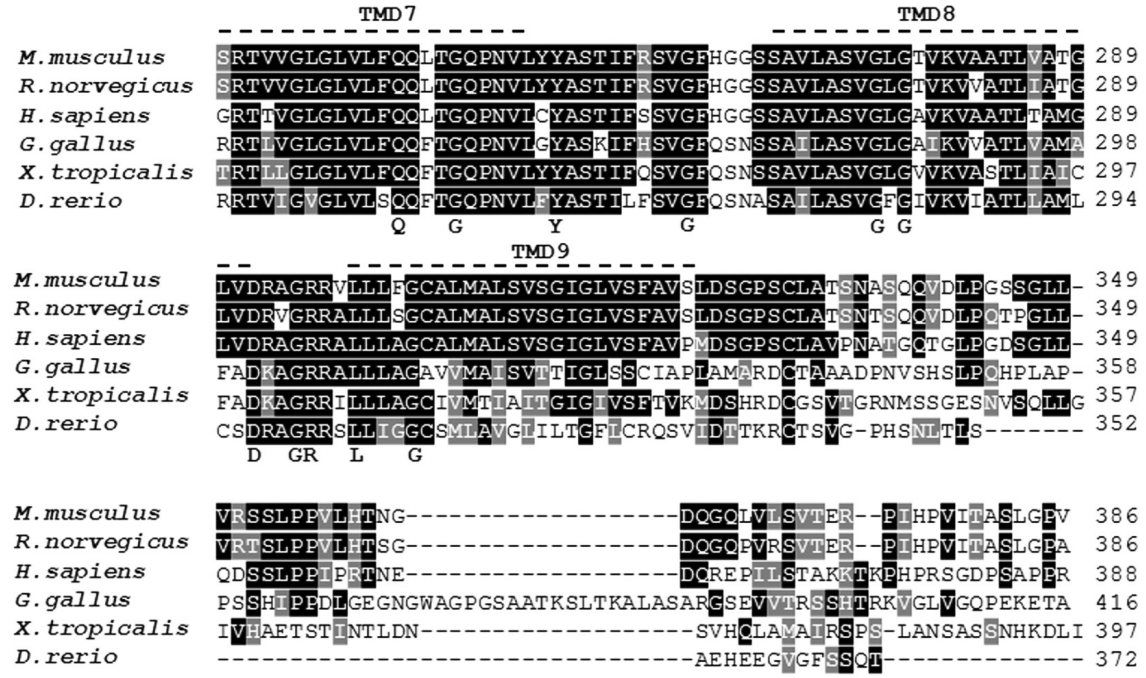
D. rerio

\section{M. musculus $R$. norvegicus H. sapiens G. gallus X. tropicalis D. rerio}

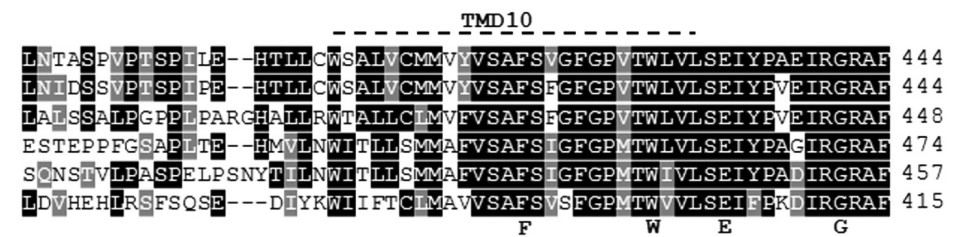

M.musculus R. norvegicus H. sapiens G.gallus X. tropicalis D. rerio

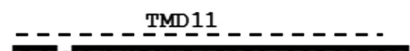
TMD 12

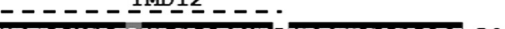
AFCSSFNWAANLFISLSFLDLIGAIGI AWTFLLYGLTAVLGLAFIYIIVPETKGOSLAE I 504 AFCNSFNWAANLFI SLSFLDLIGT IGLSWTFLLYGLTA VLGLGF IYLFVPETKGQSLAE I 508 AFCNSFNWAANIII SLSFLDL IDAIGF SWMFLLYGLMGVMAV IFIYFFVPETKGQSIEEI 534 AFCNSFNWAANLI ITITFLDVIAS IGLSWT FLLYGVVGLIA IAFIYF IPETKGQSLEE I 517 AFINCFNVGANLIVIFSFISI IDVIGLSGVFLMYGVVGIAGVVFIYLVI PETKGKSLQDI 475

M.musculus R. norvegicus H. sapiens $\begin{array}{ll}\text { G.gallus } & \text { DQQFSRKWLRE------GSGCKQRRARGASCAHGQYQRVERASSA-- } 573 \\ \text { X.tropicalis DKQFSTKRILQKRETSKGVGKRPS-------SGPPYQRIGKASPS- } & 555\end{array}$ D.rerio DRELSQTRMIHRQELCS I FQRPR-------FSPGYQRVQLTSTAT- 513 
different developmental stages. In particular, to study the expression of two s/c2a10 mRNA splice variants, a primer pair was designed to specifically amplify these isoforms. Two distinct PCR products, 727 and $550 \mathrm{bp}$, were detected at all developmental stages analyzed, including the zygote, indicating that s/c2a10 is also maternally expressed (Fig. 2A). The expression level of s/c2a10 transcripts was maintained throughout gastrulation, embryo segmentation (6-somite stage) and pharyngula periods (24-48 hpf). The s/c2a10gene was also detected during the larval period, the protruding-mouth (72 hpf), the 4-day-old and 6-dayold larval stage up to the adult fish.

Using Q-PCR, we quantified the expression levels of s/c2a10 mRNAs during embryonic development (Fig. 2B). Simultaneous detection of $e f-1 \alpha$ gene expression was used as the reference gene to normalize the expression level of s/c2a10. Quantified mRNA values were reported for s/c2a10 relative to the 1-cell stage expression level. Quantitative analysis revealed the presence of s/c2a10 maternal transcripts. The expression level of s/c2a10 mRNA was high at the one-cell stage and at the onset of mid-blastula transition ( $1 \mathrm{k}$-cell stage, $3 \mathrm{hpf}$ ); starting from this stage, the transcript levels rapidly decreased untill the 6-somite stage and remained at lower levels during subsequent stages of embryonic development (Fig. 2B).

In the adult fishes, the $s / c 2 a 10$ tissue-specific distribution was analyzed by conventional RT-PCR analysis of total RNA samples purified from several tissues and organs (Fig. 2C). Both splice variants were observed in all adult samples analyzed: liver, heart, gills, eyes, brain, spleen, swim bladder, ovary, caudal fin, testes, intestine and kidney. To assess RNA integrity, the transcript for the $\beta$-actin gene was amplified by RT-PCR from all tissues analyzed.

\section{Spatiotemporal expression of slc2a10 during development}

The spatiotemporal expression pattern of s/c2a10 in embryos from the one-cell stage to $4 \mathrm{dpf}$ larvae was investigated by wholemount ISH using a DIG-UTP-labeled probe, which recognizes both s/c2a10 mRNA splice variants. A sense probe was used in parallel control experiments that did not produce any signal (data not shown). According to RT-PCR results, ISH experiments confirmed that $s / c 2 a 10$ is expressed during early embryonic development. High levels of s/c2a10 mRNA were expressed in equal distributions from the 1-cell stage to the 1k-cell stage (Fig. $4 \mathrm{~A}, \mathrm{~F})$. During the gastrula period ( $50 \%$ epiboly and shield stage), the embryos showed the ubiquitous distribution of the s/c2a10 transcripts (Fig. $4 \mathrm{G}, \mathrm{H}$ ). In the mid-segmentation period (15 somites), transcript appeared in the otic placode and in the notochord (Fig. 4 I). At the $20-25$ somite stage, a hybridization signal was detected in the yolk syncytial layer and in the notocord (Fig. $4 \mathrm{~J}, \mathrm{~K}, \mathrm{~L}$ ). In $24 \mathrm{hpf}$ embryos, s/c2a10 mRNA was largely diffused in the cephalic region; an intense signal was also observed in the hatching gland cells and in the otic vesicles, whereas the staining in the notochord disappeared (Fig. 4M). At the longpec stage (48 hpf), s/c2a 10 transcript was mainly restricted to the pectoral fin buds, hatching gland cells, otic vesicles, lens and to the posterior tectum (Fig. $4 \mathrm{~N}, \mathrm{O}$ ). During the larval period at the protruding mouth stage (72 hpf), the signal persisted in the otic vesicles, in the pectoral fin buds, and at a low level in the posterior tectum (Fig. 4P), which is in agreement with previous reports (Tseng et al., 2009). In the swimming larva at $4 \mathrm{dpf}$, the signal

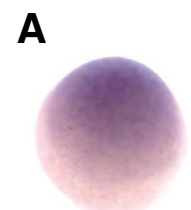

E
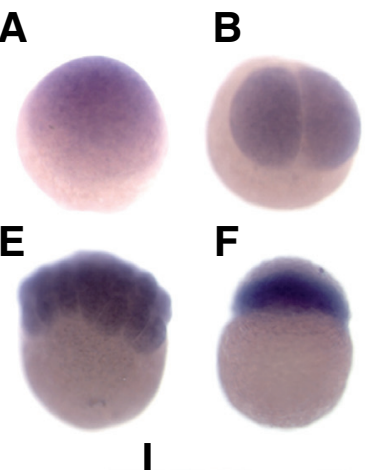

$\mathbf{F}$

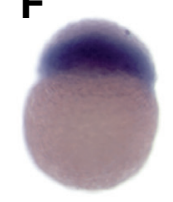

I

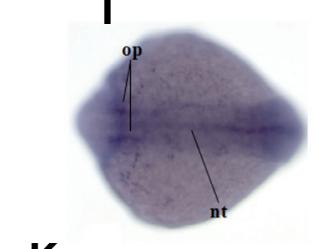

$\mathbf{K}$

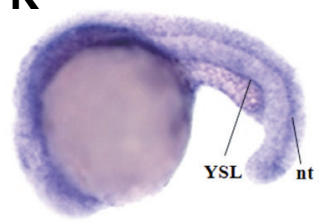

0

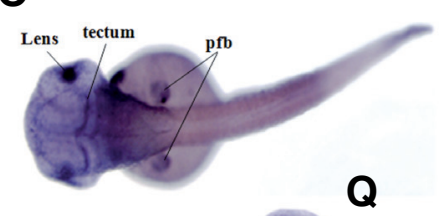

Q

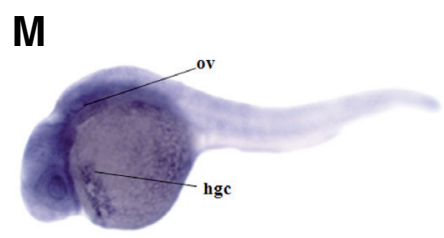

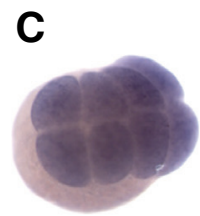

G

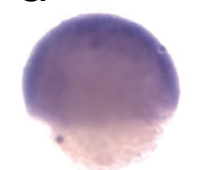

$\mathbf{J}$

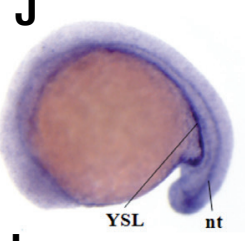

$\mathbf{L}$

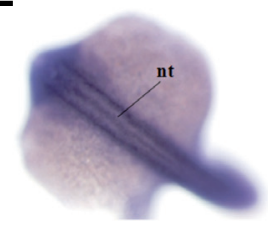

$\mathbf{N}$

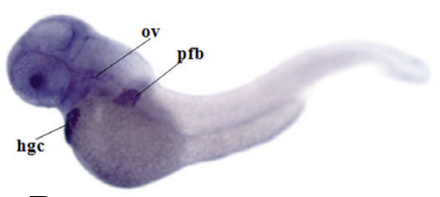

$\mathbf{P}$

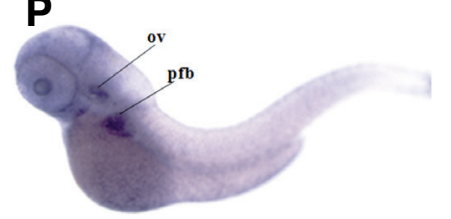

Fig. 4. Whole-mount in situhybridization analysis of s/c2a10 expression during zebrafish development. slc2a10 mRNA was ubiquitously distributed from the 1-cell stage to the $1 \mathrm{k}$-cell stage $(\mathbf{A}, \mathbf{F})$ and during the gastrula period $\mathbf{( G , H ) . ~ A t ~ t h e ~ 1 5 - s o m i t e ~ s t a g e , ~ a ~ h y b r i d i z a t i o n ~ s i g n a l ~ w a s ~}$ localized in the otic placode and notochord (I); at the 20-25 somites stage, slc2a10 transcript was also expressed in the YSL region and in the notochord $(\mathbf{J}, \mathbf{K}, \mathbf{L})$. During the pharyngula period (24-hpf), an intense signal was largely diffused in the cephalic region (M). In 48-hpf embryos $(\mathbf{N}, \mathbf{0})$, the slc2a10 mRNA was detected in the lens, posterior tectum, otic vesicles, hatching gland cells and pectoral fin buds. During the larval stage in 72-hpf embryos (P), slc2a10 expression was restricted to the otic vesicles and pectoral fins; in 4-dpf larvae (0), the staining was observed in the swim bladder structure. Abbreviations: hgc, hatching gland cells; nt, notochord; pfb, pectoral fin buds; op, otic placode; ov, otic vesicles; sb, swim bladder; YSL, yolk syncytial layer. 
appeared in the swim bladder structure (Fig. 4Q). During zebrafish embryogenesis, ISH analyses did not show a specific expression of s/c2a10 mRNA in blood vessels or in the cardiovascular system.

\section{Discussion}

In the present study, we characterized the zebrafish ortholog of the human $S L C 2 A 10$ gene and its mRNA expression pattern during development and in adult tissues.

Bioinformatics analysis indicated the presence of a unique s/c2a 10 ortholog gene located on chromosome 11 of the zebrafish genome in a region that shows conserved synteny with the $H$. sapiens chromosome $20 q 13.12$. From an evolutionary perspective, the $S L C 2 A 10$ gene structure has been remarkably conserved from fish to mammals. The zebrafish s/c2a10 is composed, like the human counterpart, of five exons with the translation initiation codon in exon 1. The deduced amino acid sequence of the Glut10 protein adopts the characteristic twelve transmembrane domain structure and contains most of the sugar/polyol transporter signatures (Joost et al., 2002; Tseng et al., 2009). Sequencing of the Glut10 coding region disclosed several nonsynonymous strain-specific polymorphisms. Multiple alignment of orthologous proteins underlined that these amino acid substitutions did not involve residues that are either evolutionarily conserved or essential for sugar transport. In contrast, all human mutations involved functionally relevant amino acids or highly conserved residues (Coucke et al., 2006; Drera et al., 2007, Callewaert et al., 2008a, Faiyaz-Ul-Haque etal., 2009; Zaidi et al., 2009; Ritelli et al., 2009).

Unlike the human $S L C 2 A 10$ gene, the s/c2a10 primary transcript in zebrafish gives rise to two specific mRNA splice variants differing in the 5'-UTR for a 177-nucleotide stretch; both encode the same polypeptide. The presence of these isoforms, not previously identified (Tseng et al., 2009), suggests the possible existence of a differential processing of zebrafish s/c2a10 premRNA. Bioinformatics analysis indicated the presence in the 177nucleotide stretch of motifs involved in the transcriptional and post-transcriptional gene expression regulation, i.e., IRES sequences and the K-box motif. Therefore, these two splice variants could provide a potential regulatory mechanism for s/c2a10gene expression at both transcriptional and post-transcriptional levels, even though their function is still unknown and requires further investigation.

During embryogenesis, both s/c2a10 mRNA splice variants were detectable in newly fertilized eggs and in adult zebrafish. Because the s/c2a 10 transcripts showed a maternal contribution as well as zygotic expression, they could be required in the early stages of zebrafish development. ISH revealed that, in early embryogenesis, s/c2a 10 was homogeneously distributed. During the segmentation stage, gene expression was restricted to the otic placode and to the notochord. In particular, at the 20-25 somite stage the s/c2a10 transcript was clearly observed in the YSL, suggesting a role for s/c2a10 in sugar transport in yolk stores. At $24 \mathrm{hpf}$, s/c2a10 showed localized expression corresponding to the encephalic region, lens, otic vesicles, posterior tectum, pectoral fin buds and hatching gland cells. The s/c2a10 mRNA detected in the otic vesicle was also observed for its murine ortholog. In particular, De Silva et al. (2006) reported that
S/c2a10 is involved in the differentiation pathway of hair cell lineage and in the development of the cochlea. These data suggest that in both animal models the gene is required for sugar transport in the cochlear tissue and could be implicated in the morphogenesis of the inner ear. The presence of s/c2a10 mRNA in the zebrafish brain could be suggestive of the existence of glucose sensing areas involved specifically in glucose homeostasis, as described in mouse (Dahlin et al., 2009). Our ISH results do not show a specific expression of s/c2a10 in the developing blood vessels of zebrafish embryos, which is probably due to a low abundance of s/c2a10mRNA. Likewise, there has been no report of specific blood vessel expression of S/c2a10in mouse embryos as evidenced by ISH data generated by the Eurexpress Consortium.

In the adult zebrafish, both s/c2a10 splicing variants were detected and showed a tissue-specific expression pattern (liver, heart, gills, eyes, brain, spleen, swim bladder, ovary, caudal fin, testes, intestine and kidney), as previously described for its human and mouse orthologs (Dawson et al., 2001; Wood et al., 2003; De Silva et al., 2006). In particular, Glut10 mRNA was expressed in tissues in which this transporter is known to play a functional role in glucose homeostasis in mammals, such as the liver and kidney (Uldry and Thorens, 2004). In addition, s/c2a10 expression in the zebrafish testes could be related to a possible requirement of glucose as an energy substrate for spermatozoa, and its expression in ovary could be related to a possible requirement in oogenesis. In agreement with the knowledge that in mammals GLUT10 is abundantly expressed in smooth musclerich organs (Wu etal., 2009), s/c2a10 was expressed in zebrafish intestine, heart and swim bladder.

Regarding GLUT10 function in ATS etiopathogenesis, two mouse models have been proposed (Callewaert et al., 2008b; Cheng et al., 2009). Both S/c2a10 mutant mice do not show the pathological features of the human disorder and therefore remain inconclusive with respect to GLUT10 function.

Recently, a new hypothesis regarding the role of GLUT10 in ATS etiology was proposed (Segade, 2010). GLUT10 may be a transporter of ascorbic acid (AA), a cofactor of the hydroxylases that modify collagen and elastin. From this perspective, the loss of GLUT10 should result in defective synthesis and abnormal assembly of these proteins. This assumption is supported by previous results (Siman et al., 1997; Maeda et al., 2000; Coucke etal., 2006). Moreover, Maeda etal., showed abnormalities of the arterial walls in knockout mice for the L-gulono- $\gamma$-lactone oxidase (Gulo), the key enzyme involved in AA synthesis. A recent study has further validated Segade's hypothesis that GLUT10 is a transporter of ascorbate (Lee et al., 2010). Indeed, Lee and colleagues demonstrated that GLUT10 primarily localizes to mitochondria in smooth muscle cells and adipocytes, facilitates transport of the oxidized form of vitamin $\mathrm{C}$ (DHA) into mitochondria and also increases cellular uptake of DHA. These authors also showed an increase of intracellular reactive oxygen species (ROS) levels that may lead to abnormal elastogenesis in the aortic smooth muscle cells of an ATS mouse model (Lee et al., 2010). This new functional role for GLUT10 may help to explain why the mutant mice do not fully mimic the human syndrome (Callewaert et al., 2008b; Cheng etal., 2009). Indeed, rodents, unlike humans, are able to synthesize $\mathrm{AA}$, and therefore S/c2a10-/- mice are less susceptible to the lack of GLUT10. Instead, zebrafish need to be 
fed a diet supplemented with AA because of the lack of a functional gulo gene. Glut10 expression in zebrafish organs and tissues observed in this study could therefore be needed for AA transport to reduce oxidative stress, particularly in muscle cells. With this taken into consideration, s/c2a 10 morpholino-mediated knockdown might disclose arterial abnormalities due to defective intracellular AA transport and improve our knowledge of the human syndrome.

\section{Materials and Methods}

\section{Bioinformatics analysis}

Nucleotide sequence assembly was performed using Sequencher version 4.9 software (www.genecodes.com). Zebrafish genomic sequences were analyzed using the UCSC Genome Browser (www.genome.ucsc.edu/) and the Ensembl Genome Browsers (www.ensembl.org). Multiple sequence alignments and phylogenetic analyses were performed using the ClustalW algorithm (www.ebi.ac.uk/ clustalw/), and the formatting of the alignment was generated using the GeneDoc program. Zebrafish Glut10 sequence was compared to those of H. sapiens (accession no. NP_110404), M. musculus (accession no. NP_569718), R. norvegicus (accession no. NP_001102433), G. gallus (accession no.XP_417383.2) and X. tropicalis(accession no. AA_22090). The presence of synteny between human and zebrafish genomic regions was evaluated using the Synteny DB (www.teleost.cs.uoregon.edu/ synteny_db/) (Catchen et al., 2009). Nucleotide and amino acid sequences were compared to the non-redundant sequence databases present at the NCBI using the BLAST algorithm. Putative TMDs were defined using the Prediction of Transmembrane helices in proteins, TMHMM server version 2.0 (www.cbs.dtu.dk/services/TMHMM-2.0). The DNA sequences at the exon/intron boundaries were determined using the Splice Site Prediction by Neural Network (www.fruitfly.org/seq_tools/ splice.html) program.

\section{Identification of zebrafish slc2a10 cDNA clones}

I.M.A.G.E (Integrated Molecular Analysis of Gene Expression) Consortium cDNA clones no. 5601920 (Genbank accession no. BM860136, 5' sequence and no. BM859903, 3' sequence) and no. 7225584 (GenBank accession no. CN015886, 5' sequence), encoding the putative zebrafish Glut10, were purchased from Geneservice (U.K.). Both cDNA clones were completely sequenced using an $A B I$ Prism 3100 automated sequencer (Applied Biosystems, Foster City, CA, USA) and vector and gene specific primers.

\section{Fish breeding and embryos/tissues collection}

The $A B$ wild-type strain of zebrafish was used for all experiments. The fishes were maintained at $28.5^{\circ} \mathrm{C}$ in $14: 10 \mathrm{~h}$ light/dark cycle following a standard procedure (Westerfield, 2000). The fertilized eggs were collected in Petri dishes and examined until the required developmental stage was reached according to established embryonic stages (Kimmel et al., 1995). For RNA extraction, zebrafish embryos and larvae were rinsed in PBS solution, frozen immediately in liquid nitrogen and stored at $-80^{\circ} \mathrm{C}$ until use. For tissue dissections from adult animals, the fishes were killed by an excess of ethyl 3-aminobenzoate methanesulfonate salt solution (Tricaine, Sigma Aldrich St. Louis, MO, USA). The tissues were quickly removed, soaked in liquid nitrogen and stored at $-80^{\circ} \mathrm{C}$ until RNA extraction.

\section{RNA preparation, RT-PCR analysis and cloning of the full length Glut10 coding sequence}

Total RNA was extracted from zebrafish embryos at different developmental stages and from several organs and tissues of adult fishes using TRIzol ${ }^{\circledR}$ protocol (Invitrogen, Carlsbad, CA, USA). The RNA concentration was determined using the NanoDrop ${ }^{\circledR}$ ND-1000 spectrophotometer
(NanoDrop Technologies, Germany). cDNA was prepared from $3 \mu \mathrm{g}$ of total RNA, using $200 \mathrm{U} / \mu \mathrm{l}$ of M-MLV reverse transcriptase (Invitrogen) in the presence of random hexamer oligonucleotides, following the manufacturer's instructions. Amplification was performed for 34 cycles $\left(60^{\circ} \mathrm{C}\right.$ annealing) following the instructions for GoTaq DNA polymerase (Promega, Madison, WI, USA) and with specific primer pairs for the s/c2a10 gene (Supplementary Table S1). In particular, to study the expression of two s/c2a10mRNA splice isoforms, the forward primer was designed upstream of the splice donor site in the $5^{\prime}$-UTR and the reverse primer was located in the coding region of s/c2a10. To assess the RNA integrity, a 679-bp fragment of the zebrafish $\beta$-actin gene (GenBank accession no. AF057040) was amplified. The PCR reactions were performed for 28 cycles using the following $\beta$-actin specific primers: $\beta$-actinfor: (5'-GTCCCTGTACGCCTCTGGTCG-3') and $\beta$-actin-rev: (5'GCCGGACTCATCGTACTCCTG-3'). To isolate the complete Glut10 coding sequence, RT-PCR-based amplification of a 1.549-bp fragment was performed on a total RNA sample from an adult AB zebrafish.

The following forward and reverse primers were used:

s/c2a10-ATG-F (5'- CATCAAAATGGGTTGTTCTGTCCTG-3') s/c2a10 TGA-R (5'-CTTCATCAGGTTGCTGTACTCGTCA-3'). A 250-ng sample of total RNA was retrotranscribed and amplified with the SuperScript III One-Step RT-PCR System (Invitrogen) using the cycling parameters provided by the manufacturer. The purified PCR product was directly cloned into the pcDNA3.1/CT-GFP-TOPO ${ }^{\text {TM }}$ vector (GFP TA Cloning ${ }^{\circledR}$ system, Invitrogen) and sequenced on both strands using Big Dye terminator v3.1 protocol on an ABI PRISM 3100 Genetic Analyzer (Applied Biosystems).

\section{Quantitative real-time RT-PCR}

Q-PCR was performed using primers designed with the Primer Express $^{\text {TM }}$ software (Applied Biosystems). The primer sequences were: s/c2a10 gene, forward: 5'-CGAAGGACATCCGAGGAAGA-3', reverse: 5'-AGACAGGCCAAATGACATCGAT-3', TaqMan probe: 5'TCATCAACTGCTTCAATGTGGGCGC-3'; ef-1 $\alpha$ gene: forward: 5'CGATTCCACCGCATTTGTAGA-3', reverse: 5'CCACGTCGACTCCGGAAA-3', TaqMan probe: 5'TCCACCACCACCGGCCATCTG-3'. The TaqMan probes (Eurofins MWG Technologies, Ebersberg, Germany) were labeled at the 5' end with the reporter dye 6-carboxy-fluorescein and at the 3' end with the quencher dye 6-carboxytetramethyl rhodamine. Amplification was performed with the $\mathrm{ABI}$ Prism 7500 sequence detection system (Applied Biosystems). The PCR was carried out with TaqMan Universal PCR Master Mix, and thermal cycling was performed according to the manufacturer's protocol (Applied Biosystems). The relative quantification (RQ) of gene expression was determined by the equation $2^{-(\Delta \Delta C t)}$. All data analysis was performed with the 7500 system SDS software, version 1.3.1 (Applied Biosystems). Threshold cycles (Ct) were standardized by $\mathrm{Ct}$ for the endogenous control ef-1 $\alpha$ mRNA.

\section{Whole-mount in situ hybridization}

Antisense and sense riboprobes were synthesized from s/c2a10 cDNA corresponding to a 1.317-bp fragment of s/c2a10 coding sequence, using SP6 and T7 RNA polymerase respectively. ISH was carried out as previously described (Thisse et al., 2008). Embryos and larvae were collected, dechorionated and incubated at $28.5^{\circ} \mathrm{C}$ at different stages. Embryos were fixed overnight in $4 \%$ PFA at $4{ }^{\circ} \mathrm{C}$, dehydrated through an ascending methanol series and stored at $-20^{\circ} \mathrm{C}$. After treatment with proteinase K (10 $\mu \mathrm{g} / \mathrm{ml}$, Roche Applied Science), the embryos were hybridized overnight at $70^{\circ} \mathrm{C}$ with DIG-labeled antisense or sense RNA probes $(300-400 \mathrm{ng} / \mathrm{\mu l})$. The staining was performed with NBT/BCIP alkaline phosphatase substrates. The stained embryos were mounted in agarose-coated dishes, photographed under an epifluorescence Leica MZ16 F stereomicroscope equipped with DFC480 digital camera and analyzed by the Leica Application Suite version 2.8.1 software (Leica Microsystems, GmbH, Wetzlar, Germany). 


\section{Acknowledgements} (2006).

This work was supported by Fondazione Cariplo "ZebraGene" Grant

\section{References}

AKHURST, R.J., (2006). A sweet link between TGFBeta and vascular disease? Nat Genet 38: 400-401.

BARRETT, M.P., WALMSLEY, A.R., GOULD, G.W. (1999). Structure and function of facilitative sugar transporters. Curr Opin Cel/ Bio/11: 496-502.

CALLEWAERT, B.L., WILLAERT, A., KERSTJENS-FREDERIKSE, W.S., DE BACKER, J., DEVRIENDT, K., ALBRECHT, B., RAMOS-ARROYO, M.A., DOCO-FENZY, M., HENNEKAM, R.C., PYERITZ, R.E., KROGMANN, O.N., GILLESSEN-KAESBACH, G., WAKELING, E.L., NIK-ZAINAL, S., FRANCANNET, C., MAURAN, P., BOOTH, C., BARROW, M., DEKENS, R., LOEYS, B.L., COUCKE, P.J., DE PAEPE, A.M. (2008a). Arterial tortuosity syndrome: clinical and molecular findings in 12 newly identified families. Hum Mutat 29: 150-158.

CALLEWAERT, B.L., LOEYS, B.L., CASTELEYN, C., WILLAERT, A., DEWINT, P., DE BACKER, J., SEDLMEIER, R., SIMOENS, P., DE PAEPE, A.M., COUCKE, P.J. (2008b). Absence of arterial phenotype in mice with homozygous s/c2a10 missense substitutions. Genesis 46: 385-389.

CATCHEN, J.M., CONERY, J.S., POSTLETHWAIT, J.H. (2009). Automated identification of conserved synteny after whole genome duplication. Genome Research 19: 1497-1505.

CHENG, C.H., KIKUCHI, T., CHEN, Y.H., SABBAGHA, N.G., LEE, Y.C., PAN, H.J., CHANG, C., CHEN, Y.T. (2009). Mutations in the SLC2A10 gene cause arterial abnormalities in mice. Cardiovascular Research 81: 381-388.

COUCKE, P.J., WILLAERT, A., WESSELS, M.W., CALLEWAERT, B., ZOPPI, N., DE BACKER, J., FOX, E., MANCINI, G.M., KAMBOURIS, M., GARDELLA, R., FACCHETTI, F., WILLEMS, P.J., FORSYTH, R., DIETZ, H.C., BARLATI, S., COLOMBI, M., LOEYS, B., DE PAEPE, A. (2006). Mutations in the facilitative glucose transporter GLUT10 alter angiogenesis and cause arterial tortuosity syndrome. Nat Genet 38: 452-457.

DAHLIN, A., ROYALL, J., HOHMANN, J.G., WANG, J. (2009). Expression Profiling of the Solute Carrier Gene Family in the Mouse Brain. $J$ Pharmacol Exp Ther 329: $558-570$.

DAWSON, P.A., MYCHALECKYJ, J.C., FOSSEY, S.C., MIHIC, S.J., CRADDOCK, A.L., BOWDEN, D.W. (2001). Sequence and functional analysis of GLUT10: a glucose transporter in the Type 2 diabetes-linked region of chromosome 20q1213.1. Mol Genet Metab 74: 186-199.

DE SILVA, M.G., HILDEBRAND, M.S., CHRISTOPOULOS, H., NEWMAN, M.R., BELL, K., RITCHIE, M., SMYTH, G.K., DAHL, H.H. (2006). Gene expression changes during step-wise differentiation of embryonic stem cells along the inner ear hair cell pathway. Acta Otolaryngo/ 126: 1148-1157.

DRERA, B., GUALA, A., ZOPPI, N., GARDELLA, R., FRANCESCHINI, P., BARLATI, S., COLOMBI, M. (2007). Two novel SLC2A10GLUT10 mutations in a patient with arterial tortuosity syndrome. Am J Med Genet 143: 216-218.

FAIYAZ-UL-HAQUE, M., ZAIDI, S.H., AL-SANNA, N., ALSWAID, A., MOMENAH, T., KAYA, N., AL-DAYEL, F., BOUHOAIGAH, I., SALIEM, M., TSUI, L.C., TEEBI, A.S. (2009). A novel missense and a recurrent mutation in SLC2A10 gene of patients affected with arterial tortuosity syndrome. Atherosclerosis203: 466-471.

GURYEV, V., KOUDIJS, M.J., BEREZIKOV, E., JOHNSON, S.L., PLASTERK, R.H.A., VAN EEDEN, F.J.M., CUPPEN, E. (2006). Genetic variation in the zebrafish. Genome Research 16: 491-497.
JOOST, H.G., BELL, G.I., BEST, J.D., BIRNBAUM, M.J., CHARRON, M.J., CHEN Y.T., DOEGE, H., JAMES, D.E., LODISH, H.F., MOLEY, K.H., MOLEY, J.F., MUECKLER, M., ROGERS, S., SCHÜRMANN, A., SEINO, S., THORENS, B. (2002). Nomenclature of the GLUT/SLC2A family of sugar/polyol transport facilitators. Am J Physiol Endocrinol Metab 282: 974-976.

JOOST, H.G. and THORENS, B. (2001). The extended GLUT-family of sugar/ polyol transport facilitators: nomenclature, sequence characteristics, and potential function of its novel members. Mo/ Membr Bio/ 18: 247-256.

KIMMEL, C.B., BALLARD, W.W., KIMMEL, S.R., ULLMANN, B., SCHILLING, T.F (1995). Stages of embryonic development of the zebrafish. Dev Dynam 203: 253-310.

LEE, Y.C., HUANG, H.Y., CHANG. C.J., CHENG, C.H., CHEN, Y.T. (2010). Mitochondrial GLUT10 facilitates dehydroascorbic acid import and protects cells against oxidative stress: mechanistic insight into arterial tortuosity syndrome. Hum Mol Genet 19: 3721-3733.

MAEDA, N., HAGIHARA, H., NAKATA, Y., HILLER, S., WILDER, J., REDDICK, R. (2000). Aortic wall damage in mice unable to synthesize ascorbic acid. ProcNat/ Acad Sci. USA 97: 841-846.

MCVIE-WYLIE, A.J., LAMSON, D.R., CHEN, Y.T. (2001). Molecular cloning of a novel member of the GLUT family of transporters, SLC2A10 (GLUT10), localized on chromosome 20q13.1: a candidate gene for NIDDM susceptibility. Genomics 72: 113-117.

PARANT, J.M., GEORGE, S.A., HOLDEN, J.A., YOST, H.J. (2010). Genetic modeling of Li-Fraumeni syndrome in zebrafish. Dis Mode/ Mech 3: 45-56.

RITELLI, M., DRERA, B., VICCHIO, M., PUPPINI, G., BIBAN, P., PILATI, M., PRIOLI, M.A., BARLATI, S., COLOMBI, M. (2009). Arterial tortuosity syndrome in two Italian paedriatric patients. Orphanet $J$ Rare Dis 4: 20.

SEGADE, F. (2010). Glucose transporter 10 and arterial tortuosity syndrome: The vitamin C connection. FEBS letters 584: 2990-2994.

SIMAN, C.M. and ERIKSSON, U.J. (1997). Vitamin C supplementation of the maternal diet reduces the rate of malformation in the offspring of diabetic rats. Diabetologia 40: 1416-1424.

THISSE, C. and THISSE, B. (2008). High-resolution in situhybridization to wholemount zebrafish embryos. Nature Prot 3: 59-69.

TSENG, Y.C., CHEN, R.D., LEE, J.R., LIU, S.T., LEE, S.J., HWANG, P.P. (2009) Specific Expression and Regulation of Glucose Transporters in Zebrafish lonocytes. Am J Physiol Regul Integr Comp Physio/297: 275-290.

ULDRY, M. and THORENS, B. (2004). The SLC2 family of facilitated hexose and polyol transporters. Eur J Physio/447: 480-489.

WESTERFIELD, M. (2000). The zebrafish book: A guide for the laboratory use of zebrafish. University of Oregon Press, Eugene.

WOOD, I.S., HUNTER, L., TRAYHURN, P. (2003). Expression of Class III facilitative glucose transporter genes (GLUT-10 and GLUT-12) in mouse and human adipose tissues. Biochem Biophys Res Commun 308: 43-49.

WU, C., OROZCO, C., BOYER, J., LEGLISE, M., GOODALE, J., BATALOV, S., HODGE, C.L., HAASE, J., JANES, J., HUSS, J.W. ${ }^{\text {rd }}$, SU, A.I. (2009). BioGPS: an extensible and customizable portal for querying and organizing gene annotation resources. Genome Biology 10: R130.

ZAIDI, S.H., MEYER, S., PELTEKOVA, V.D., LINDINGER, A., TEEBI, A.S., FAIYAZ-UL-HAQUE, M. (2009). A novel non-sense mutation in the $S L C 2 A 10$ gene of an arterial tortuosity syndrome patient of Kurdish origin. Eur J Pediatr 168: 867-870

ZHAO, F.Q. and KEATING, A.F. (2007). Functional properties and genomics of glucose transporters. Current Genomics 8: 113-128. 


\section{Further Related Reading, published previously in the Int. J. Dev. Biol.}

Xenopus glucose transporter 1 (xGLUT1) is required for gastrulation movement in Xenopus laevis Keiko Suzawa, Akira Yukita, Tadayoshi Hayata, Toshiyasu Goto, Hiroki Danno, Tatsuo Michiue, Ken W. Cho and Makoto Asashima Int. J. Dev. Biol. (2007) 51: 183-190

TBX1, a DiGeorge syndrome candidate gene, is inhibited by retinoic acid Lifeng Zhang, Tao Zhong, Yuexiang Wang, Qiu Jiang, Houyan Song and Yonghao Gui Int. J. Dev. Biol. (2006) 50: 55-61

Cdx2 specifies the differentiation of morphological as well as functional absorptive enterocytes of the small intestine Hiroyuki Mutoh, Kiichi Satoh, Hiroto Kita, Hirotsugu Sakamoto, Hiroko Hayakawa, Hironori Yamamoto, Norio Isoda, Kiichi Tamada, Kenichi Ido and Kentaro Sugano

Int. J. Dev. Biol. (2005) 49: 867-871

Embryonic expression of Xenopus SGLT-1L, a novel member of the solute carrier family 5 (SLC5), is confined to tubules of the pronephric kidney

Samer R Eid, Anne Terrettaz, Katsumi Nagata and André W Brändli

Int. J. Dev. Biol. (2002) 46: 177-184

Astroglia-microvessel relationship in the developing human telencephalon

D Virgintino, E Maiorano, M Errede, A Vimercati, P Greco, L Selvaggi, L Roncali and M Bertossi

Int. J. Dev. Biol. (1998) 42: 1165-118

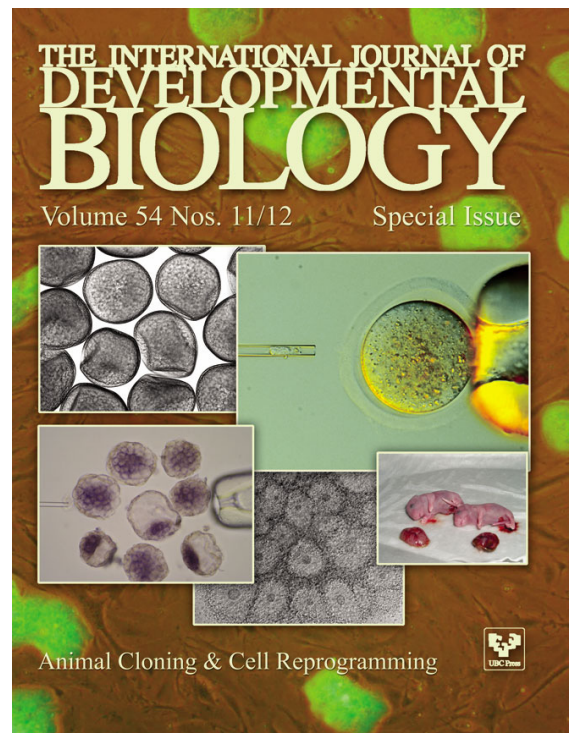

5 yr ISI Impact Factor $(2009)=3.253$

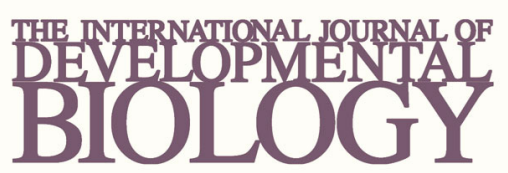

Volume 54 Nos. 6/7
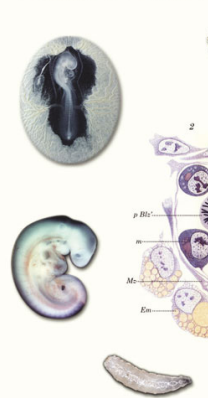

Developmental Hematopoiesis

Special Issue

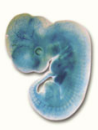

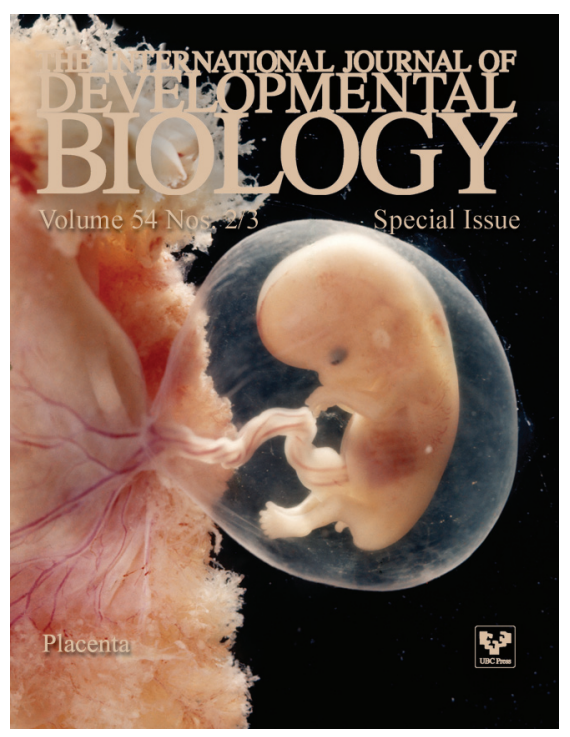

